\title{
La dinámica del territorio en la conurbación Concepción-Talcahuano: huellas urbanas para una interpretación de las transformaciones ambientales durante la segunda mitad del siglo $\mathrm{XX}^{1}$
}

\author{
Enrique Aliste Almuna ${ }^{2}$, Angie Almendras Varela ${ }^{3}$ y Miguel Contreras \\ $\mathrm{AlonsO}^{4}$
}

\section{RESUMEN}

Sin ahondar en los efectos ambientales en sí mismos, se busca aquí dar una mirada en perspectiva a los procesos urbanos y las huellas que estos que van dejando en la impronta de la ciudad. A partir de ello, busca trazar algunas líneas sobre posibles hipótesis en torno a cómo, desde esta mirada, los discursos mismos del desarrollo pueden reproblematizarse y de este modo buscar también formas diferentes para entender y proponer, en el futuro, planes y proyectos que usen como eje discursivo las ideas sobre desarrollo, desarrollo sustentable, ordenamiento y planificación territorial sustentable y sustentabilidad en general. De esta forma, se entregan algunos resultados a ser discutidos sobre los procesos urbanos y las eventuales consecuencias de estas transformaciones en la segunda mitad del siglo XX en el Gran Concepción.

Palabras clave: Huellas territoriales, desarrollo y desarrollo sustentable, geografía social.

\begin{abstract}
Without delving a specific view on the environmental effects, we are searching for a perspective to look the urban processes and the traces they have left on the city marks. From this, it have been drawn some lines of possible hypotheses about how, from this perspective, the development discourses can be re-problematized and thus seek ways to understand and propose future plans and projects using, as discursive axis, the ideas about development, sustainable development, sustainable land use management and planning and sustainability in general. In this way, some results are delivered to be discussed about urban processes and the possible consequences of these changes in the second half of the twentieth century in the city of Concepción (Chile).
\end{abstract}

Key words: Territorial traces, development and sustainable development, social geography.

\footnotetext{
Proyecto FONDECYT 1090248. Huellas y dinámicas territoriales: imaginarios del desarrollo y prácticas de transformación del ambiente en el Gran Concepción. Artículo recibido el 20 de mayo de 2011, aceptado el 16 de enero de 2012 y corregido el 16 de junio de 2012.
}

2 Departamento de Geografía, Universidad de Chile (Chile).E-mail: ealiste@uchilefau.cl

3 Servicio Nacional de Turismo (Chile). E-mail: aalmendras@sernatur.cl

4 Departamento de Geografía, Universidad de Chile (Chile). E-mail: mcalonso@uchilefau.cl 
Los procesos que han caracterizado la dinámica del territorio durante la segunda mitad del siglo XX en el Gran Concepción, han estado fuertemente marcados por su impronta asociada a la actividad industrial. Con la puesta en marcha de la CORFO y de los planes para estimular la industria en el país, la actual Región del Biobío y en particular la actual provincia de Concepción ha sido muy atractiva para el desarrollo de actividades económicas ligadas a la manufactura.

La actividad portuaria, sus condiciones de localización en general, además de los estímulos económicos recibidos desde los distintos gobiernos a partir de 1939 y en especial en $1942^{5}$, comenzaron a crear las condiciones para desarrollar en la zona una prolífica actividad industrial que significó importantes incrementos para el PIB nacional y regional, además de incrementos relevantes de la población que comienza a llegar a la zona (Hernández, 1983; Aliste y Almendras, 2010). Todos estos aspectos, que sin duda comienzan a hablar de una prosperidad a la luz de las estrategias de desarrollo, paulatinamente comienzan a mostrar una serie de efectos indeseados que en la economía ambiental se denominan "externalidades negativas del desarrollo" (Field, 2003).

Importantes trabajos se han realizado sobre el Gran Concepción y su dinámica espacial. Obras indispensables son las de Hernández (1983), Mazzei De Grazia y Pacheco (1985), Oliver y Zapata (1950), Campos (1979), Moreno (1964), Azócar y Sanhueza (2000), Pacheco (1997), entre muchos otros. Un reciente trabajo compilatorio de Pérez e Hidalgo (2010) logra distinguir tipos de trabajos realizados en la materia y que son agrupados en: i) un grupo de estudios histó-

\footnotetext{
5 En 1942 se constituye la "Comisión Gubernativa" para el estudio del proyecto siderúrgico nacional. Esta comisión, creada mediante el Decreto de Fomento $\mathrm{N}^{\circ} 1420$ del 15 de julio de 1942, estaba encabezada por el ministro de Economía y Comercio y por el ministro de Fomento. La integraban, además, los representantes de la industria siderúrgica, miembros del Instituto de Ingenieros de Chile, del Instituto de Ingenieros de Minas de Chile, además de privados que habían planteado la necesidad de llevar a cabo un proyecto siderúrgico en Chile (Echeñique y Rodríguez, 1990: 87).
}

rico-monográficos clásicos y que marcan una suerte de inicio a una tradición en la forma de mirar la ciudad; ii) un segundo grupo de estudios en donde se suma lo realizado por una publicación periódica de arquitectura (Revista AUCA), más los trabajos de corte geohistórico (Hernández, 1983), de carácter estratégico territorial (Leone, 1993) y con un perfil interdisciplinar (Rojas et al., 2006); iii) un tercer grupo de estudios de carácter más heterogéneo y reciente, se aboca a las transformaciones de la ciudad en una perspectiva urbano-económica, demográfica, socioterritorial y de identidad (Rojas et al., 2009; Pérez y Salinas, 2007, 2009; Baeriswyl, 2009; Azócar y Sanhueza, 1999; Lira, 2001); iv) un cuarto grupo de estudios, finalmente, se enfoca a los temas propiamente referidos a la sustentabilidad urbana, con una mirada a las consecuencias ecosistémicas de los procesos de urbanización (Pauchard et al., 2006; Romero et al., 2009; Smith, 2009; Mardones y Vidal, 2001).

Internacionalmente, la discusión sobre el desarrollo de las áreas metropolitanas es extensa y abundante. Sin embargo, en lo que respecta a ciudades latinoamericanas, los trabajos de Borsdorf (2003), Janoschka (2002), Bähr y Mertins (1993) pueden señalarse entre los principales. Si bien en cada uno de ellos hay propuestas que permiten entender procesos, pasos y morfología del crecimiento urbano en las ciudades latinoamericanas, es cierto que la concepción del desarrollo responde al modelo clásico de mirarlo como referencia más que como categoría de análisis que sea discutida.

Tal como se puede apreciar en la revisión de la literatura anterior, la problematización misma del concepto de desarrollo no es un elemento que se encuentre presente ni muy discutido; por el contrario, parece aceptarse y consolidarse en una parte importante de estos. Si bien en la mayoría se problematizan las consecuencias del proceso de urbanización y sus características, en ellos se logra subentender también un desarrollo como concepto pertinente y legítimo en sentido estricto, sin mirar en él una oportunidad que desde una dimensión crítica permita rediscutir los procesos observados. En este trabajo se quiere dar una mirada en donde el concepto de desarrollo y su discurso en sí pueda discutirse y problematizarse, en tanto es el que 
argumenta las transformaciones radicales de la estructura de un territorio, dejando en especial una indeleble huella de deterioro ambiental.

Por lo anterior, en el presente artículo se busca exponer, a partir de un análisis espacial centrado en las tendencias del proceso y los testimonios de las transformaciones espaciales, algunas de las consecuencias que territorialmente parecen estar incidiendo de manera importante en las condiciones ambientales existentes en la zona de estudio.

Para estos propósitos, el presente documento expone una exploración en torno a las transformaciones espaciales ocurridas durante la segunda mitad del siglo XX $y$, desde allí, discutir en torno a las ideas anteriormente planteadas.

La discusión se desarrollará a partir de la observación de dos procesos estrechamente vinculados: primero cuantificando los cambios en la extensión de la superficie urbana en el período señalado $y$, asociado a lo anterior, examinando los cambios en el uso del suelo que allí ocurre.

\section{Explorar las dinámicas territoriales: pasos y procesos metodológicos}

La metodología aplicada contempló el uso de técnicas de fotointerpretación basadas en los criterios planteados por Chuvieco (2002), que permiten la clasificación de estructuras urbanas de acuerdo a elementos tales como color, rugosidad, forma y tamaño, entre otros. Dichas técnicas permiten obtener información muy relevante con respecto a la identificación de límites urbanos, infraestructura y tipos de uso, aprovechando el mayor nivel de detalle de las fotografías aéreas en comparación con las imágenes satelitales disponibles.

En específico, se trabajó con fotografías aéreas de los períodos $1943^{6}$ (Vuelo Trime-

6 El vuelo Trimetrogón del año 1943, solo muestra el área urbana de Concepción; no existen líneas de trogón - IGM, 1:40.000), 1955 (Vuelo Hycon - IGM, 1:70.000), 1961 (Vuelo O.E.A - IGM, $1: 20.000), 1976$ y 1980 (SAF, $1: 30.000$ ), 1998 (CONAMA, 1:115.000); y con la imagen satelital 2006 (Quickbird - Google Earth). Éstas se digitalizaron y se procesaron mediante la plataforma ArcGIS 9.3. En el proceso de fotointerpretación se contempló la delimitación de las áreas urbanas de Concepción, Talcahuano y Hualpén ${ }^{7}$ para cada período. Junto con ello, se realizó una fotointerpretación de los usos y coberturas de suelo existentes, lo que permitió identificar las transformaciones espaciales más importantes del área de estudio. Los criterios empleados involucran el análisis de formas, tonos, rugosidad, luminosidad y la identificación de patrones de construcción, trama urbana y de espacios vacíos, que permitieron identificar cómo la ciudad creció a través del tiempo en base a la consolidación de las viviendas construidas y el desarrollo de equipamiento en ellas.

Debido a que la interpretación de usos de suelo dentro del área urbana es significativamente compleja, al no existir necesariamente una relación causal directa entre forma y función urbana (lo que se hace más patente en períodos más antiguos), se debió complementar la información con otro tipo de fuentes. Entre ellas destaca el análisis de los Planos Reguladores Comunales ${ }^{8}$ del área de estudio, así como bibliografía relacionada con los usos de suelo existentes en un período determinado.

Para el análisis de estas transformaciones espaciales, se tomó como base la clasificación de Usos y Coberturas de Suelo desarrollada por Smith (2007) y Moscoso (2007).

vuelo disponibles para las áreas urbanas de Talcahuano ni Hualpén.

7 Se considera Hualpén, pues dicha comuna formaba parte de la comuna de Talcahuano hasta el año 2004. El Decreto Ley No 19.936 (D.O. 13 de marzo de 2004) da origen a la comuna de Hualpén.

8 Los planos reguladores usados fueron los siguientes: para Concepción se usaron los instrumentos de 1948, 1962, 1982 y el vigente del año 2004. En el caso de Talcahuano se emplearon los instrumentos de 1960, 1982 y 2005. En el caso de Hualpén se utilizó el PRC del año 2004 y se complementó dicha información con la existente en el Plan Regulador Metropolitano de 2003. 
Junto con ello, se consideraron las tipologías de uso y función definidos por normativas complementarias de la planificación urbana chilena vigente, como es el caso de la circular DDU $N^{\circ} 55$ del Ministerio de Vivienda y Urbanismo.

Con estos antecedentes se generó una clasificación estructurada en cuatro grandes grupos de usos: Urbano, Agrícola, Vegetación Natural e Hidrología, las que comprendían subcategorías que se volvían más específicas de acuerdo al nivel de detalle que pudiera conseguirse con la fotointerpretación. Un ejemplo de las subcategorías del uso Urbano (que resulta clave para el análisis) se muestra en la Figura $N^{\circ} 1$.

Cuadro $\mathrm{N}^{\circ} 1$

Algunos elementos considerados en la clasificación de uso de suelo urbano

\begin{tabular}{|c|c|c|}
\hline Nivel 1 & Nivel 2 & Nivel 3 \\
\hline \multirow{4}{*}{$\begin{array}{l}\text { Urbano: } \\
\text { Superficie cons- } \\
\text { truida dentro de un } \\
\text { continuo asociada } \\
\text { a una red de trans- } \\
\text { porte }\end{array}$} & $\begin{array}{l}\text { Residencial: áreas urbanas con } \\
\text { variados tamaños y formas de las } \\
\text { construcciones. Presencia de co- } \\
\text { lores oscuros o plateados en los } \\
\text { techos. En ciertos casos se observa } \\
\text { vegetación interior en patios y pis- } \\
\text { cinas pequeñas. }\end{array}$ & $\begin{array}{l}\text { Asentamientos irregulares: Viviendas } \\
\text { emplazadas en zonas sin conexión } \\
\text { estructural con el damero original, ni } \\
\text { cualquier otro plano que se desarrolle } \\
\text { en el área. Las viviendas muestran una } \\
\text { configuración desigual y relativamente } \\
\text { caótica en el espacio. } \\
\text { Conjunto de vivienda: viviendas insertas } \\
\text { dentro de manzanas, bien estructuradas, } \\
\text { con patrones de regularidad espacial. }\end{array}$ \\
\hline & $\begin{array}{l}\text { Equipamiento: áreas con cons- } \\
\text { trucciones de variada extensión, } \\
\text { insertas en la trama urbana en las } \\
\text { que se lleva a cabo algún tipo de } \\
\text { servicio. Fueron identificadas con } \\
\text { la ayuda de Google Earth e infor- } \\
\text { mación secundaria. }\end{array}$ & $\begin{array}{l}\text { Educacional: Colegios, Universidades, } \\
\text { etc. } \\
\text { Salud: Hospitales, Consultorios, etc. } \\
\text { Seguridad: Cuarteles de Carabineros y } \\
\text { Bomberos. } \\
\text { Cementerio } \\
\text { Militar: Regimientos o establecimientos } \\
\text { militares. }\end{array}$ \\
\hline & $\begin{array}{l}\text { Recreacional: se observan como } \\
\text { sectores abiertos insertos en la } \\
\text { ciudad, la mayor parte de ellos } \\
\text { con vegetación interior e infraes- } \\
\text { tructura asociada a la entretención }\end{array}$ & $\begin{array}{l}\text { Áreas verdes: superficies con vegetación } \\
\text { interior del continuo urbano, se consi- } \\
\text { deran parques, plazas, centros recrea- } \\
\text { cionales, etc. } \\
\text { Estadios y canchas } \\
\text { Hipódromo }\end{array}$ \\
\hline & $\begin{array}{l}\text { Industrial: áreas con superficie } \\
\text { artificial sin vegetación que ocu- } \\
\text { pe la mayoría del área, contiene } \\
\text { grandes construcciones. } \\
\text { Fábricas e instalaciones antrópicas } \\
\text { (como viveros) que tienen relación } \\
\text { con el procesamiento de alguna } \\
\text { materia prima. }\end{array}$ & $\begin{array}{l}\text { Industria liviana: se consideran manu- } \\
\text { facturas de menor tamaño y algunas zo- } \\
\text { nas de bodegaje asociado a las grandes } \\
\text { industrias. } \\
\text { Industria pesada: grandes empresas que } \\
\text { ocupan grandes extensiones de terre- } \\
\text { no, se distinguen por la infraestructura } \\
\text { asociada (tanques, grandes chimeneas, } \\
\text { etc.). }\end{array}$ \\
\hline
\end{tabular}

Fuente: Elaboración propia. 
Posteriormente, y mediante el uso de Sistema de Información Geográfica (SIG), fue posible realizar el análisis espacial que permitió generar indicadores de superficie y definir tasas de crecimiento y evolución para el período analizado.

El siguiente paso de la investigación fue identificar períodos de mayor regularidad con el fin de vincularlos a los grandes períodos de desarrollo urbano. De esta manera se realizó una interpretación de antecedentes que permitiera establecer una relación entre las formas y cambios urbanos, vinculado a hechos o hitos urbanos relevantes.

\section{La dinámica territorial como testimonio ambiental: huellas y transformaciones para discutir una geografía social de la ciudad}

\section{Crecimiento demográfico y urbano}

Los antecedentes expuestos en la Figura $N^{\circ} 1$ muestran que entre los años 1907 y 2002 la población del Gran Concepción creció significativamente, sobre todo en el período 1940-1992, tendiendo a un menor crecimiento en el último período intercensal. Para el año 2002, se estima que la población del Gran Concepción superó los 660.000 habitantes, y hoy en día se estima que la población del Área Metropolitana del Gran Concepción (AMC) podría superar el millón de habitantes (Pérez e Hidalgo, 2010). El descenso observado en los últimos decenios en la población de Concepción, se debe a la creación de las nuevas comunas de San Pedro de la Paz y Chiguayante.

Al analizar el comportamiento demográfico intercensal y especialmente comparando con el Gran Santiago (Cuadro $N^{\circ} 2$ ), se muestra un crecimiento que se acelera algo más tardíamente (1952), dura un poco más (hasta 1982), deteniéndose bruscamente en la década de 1980. En este sentido, se puede identificar el incremento más importante en el período 1952-1982, que como hipótesis podría estar asociado al desarrollo del área industrial iniciada con la instalación y puesta en operaciones de la Siderúrgica Huachipato de la Compañía de Aceros del Pacífico (CAP)

Figura $\mathrm{N}^{\circ} 1$

Evolución demográfica del Gran Concepción

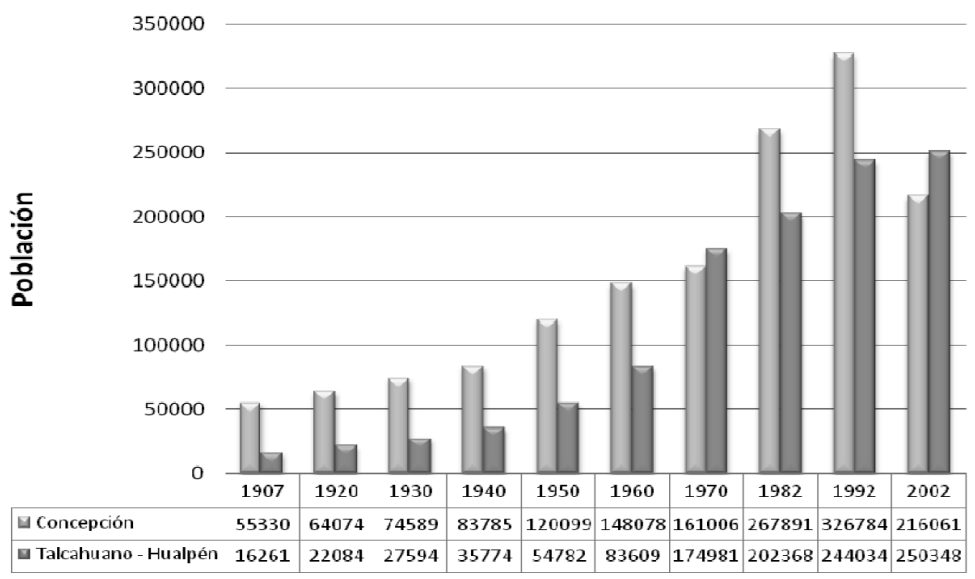

Fuente: Instituto Nacional de Estadísticas, varios años. 
Cuadro $\mathrm{N}^{\circ} 2$

Tasas de crecimiento demográfico del Gran Concepción y el Gran Santiago (1920-2002)

\begin{tabular}{|c|r|r|}
\hline Período & Tasa Crecimiento Gran Concepción & Tasa Crecimiento Gran Santiago \\
\hline $1920-1930$ & 1,3 & 3,3 \\
$1930-1940$ & 1,7 & 3,2 \\
$1940-1952$ & 1,8 & 3,2 \\
$1952-1960$ & 3,5 & 3,3 \\
$1960-1970$ & 4,0 & 4,1 \\
$1970-1982$ & 3,3 & 1,8 \\
$1982-1992$ & 2,5 & 4,1 \\
$1992-2002$ & 2,0 & 1,9 \\
\hline
\end{tabular}

Fuente: Instituto Nacional de Estadísticas, 1992; 2002.

en la década del 50. El descenso posterior puede ser multicausal, asociado tanto a la cuarta etapa de la transición demográfica que se manifestó en todo el país (con bajas tasas de crecimiento general), así como a la crisis industrial y económica de los años 1980, que afectó significativamente a Concepción.

Ahora bien, es interesante observar cómo el aumento de población durante el período analizado posee una relación directamente proporcional con el aumento en superficie del área urbana. En efecto, de una superficie inicial cercana a las 800 hectáreas en 1943, la ciudad llega a tener 6.481 hectáreas en el año 2006 (Figura $\mathrm{N}^{\circ}$ 2). Este crecimiento es muy significativo, sin embargo en términos absolutos, se encuentra muy por debajo del crecimiento del área urbana experimentada por el Área Metropolitana de Santiago ${ }^{9}$.

Por su parte, en el Figura $N^{\circ} 3$ se muestra cómo la conurbación se ha extendido a través del tiempo (1943-2006), siguiendo diversos patrones de ocupación. En este contexto, se distingue el crecimiento por "acreción"10,

9 De acuerdo con los datos presentados por Romero y Sarricolea (2006), el año 1975 la ciudad de Santiago tenía 33.685 ha, cifra que aumentó en 1989 a 39.542 ha, posteriormente en 1998, la ciudad presentó un total de 51.735 ha y al año 2004 alcanzó las 62.235 ha.

10 Crecimiento por "acreción" se caracteriza por la adherencia de nuevas áreas sobre los bordes y límites el cual se desarrolla por la adherencia de nuevas áreas urbanas a los límites de las ciudades y que tiene una mayor participación en la ciudad de Concepción. Otra modalidad es el crecimiento "tentacular"11, en donde las ciudades se extienden siguiendo las vías de transporte, el que se presenta con fuerza en el sureste de Talcahuano, donde actualmente se ubican diversas poblaciones en la ruta que conecta Concepción con Talcahuano. Finalmente se distingue el crecimiento de tipo "salto de rana"12, presente en Hualpén y entre Concepción-Penco.

\footnotetext{
urbanos, como lo plantea Ducci (1998) en "La ciudad se amplía" (Romero et al., 2001; Molina, 2007; Smith, 2007; Capel, 2002).

${ }^{11}$ Crecimiento "tentacular" corresponde a aquel que se desarrolla a través de los principales ejes de transporte, ya sean carreteras, líneas ferroviarias, etc. (Romero et al., 2001; Molina, 2007; Smith, 2007; Capel, 2002).

12 Crecimiento tipo "salto de rana" es aquel desarrollo de unidades urbanas fuera de los límites establecidos; pueden ser de distinto tamaño en cuanto a superficie construida. Surgen como respuesta habitacional para la población de menos recursos (por abaratamiento de la tierra) o por la ocupación de áreas aisladas exclusivas para aquellos que poseen más recursos. Deriva también de una estrategia de crecimiento que ocupa primero el suelo más alejado y barato y luego revaloriza los espacios intermedios. Hay colonización y ocupación de ciertos espacios y luego relleno de los intersticios urbanos (Romero et al., 2001; Molina, 2007; Smith, 2007; Capel, 2002).
} 
TALCAHUANO: HUELLAS URBANAS PARA UNA INTERPRETACIÓN DE LAS TRANSFORMACIONES AMBIENTALES DURANTE LA SEGUNDA MITAD DEL SIGLO XX

Figura $\mathrm{N}^{\circ} 2$

Evolución de la superficie en la conurbación Concepción - Talcahuano (en hectáreas según año).

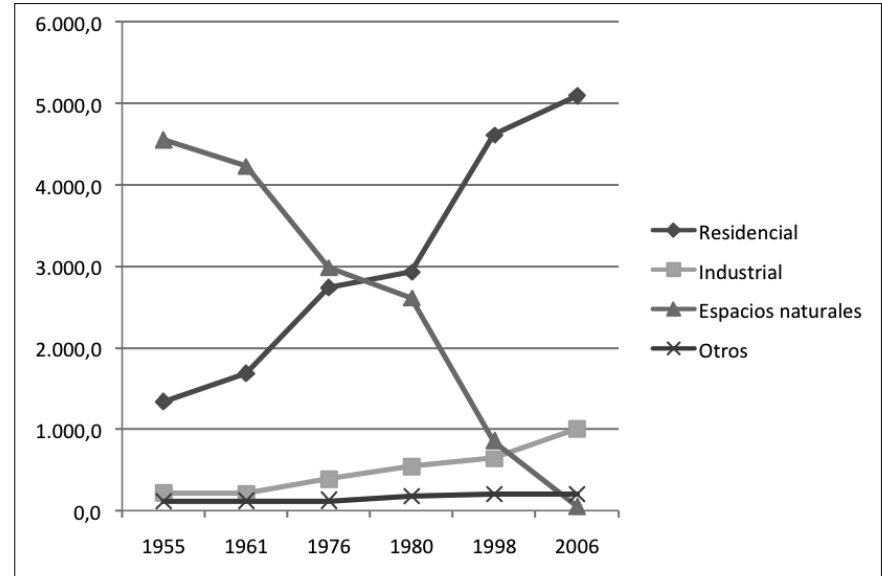

Fuente: Aliste y Almendras, 2010.

Figura $\mathrm{N}^{\circ} 3$

Secuencia evolutiva de la conurbación Concepción-Talcahuano, 1943-2006.
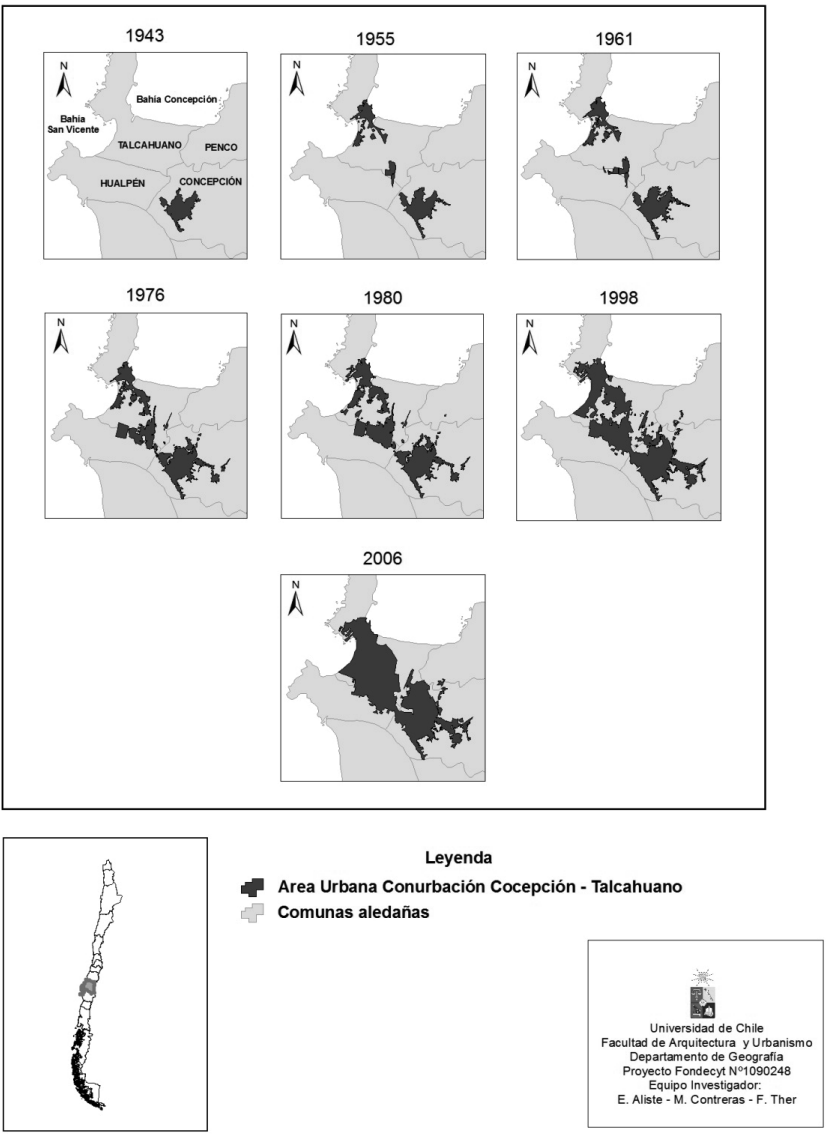

Fuente: Elaboración propia basada en vuelos Trimetrogón (1943), Hycon (1955), OEA (1976), SAF (1980), CONAMA (1998) y Quickbird-Google Earth (2006). 


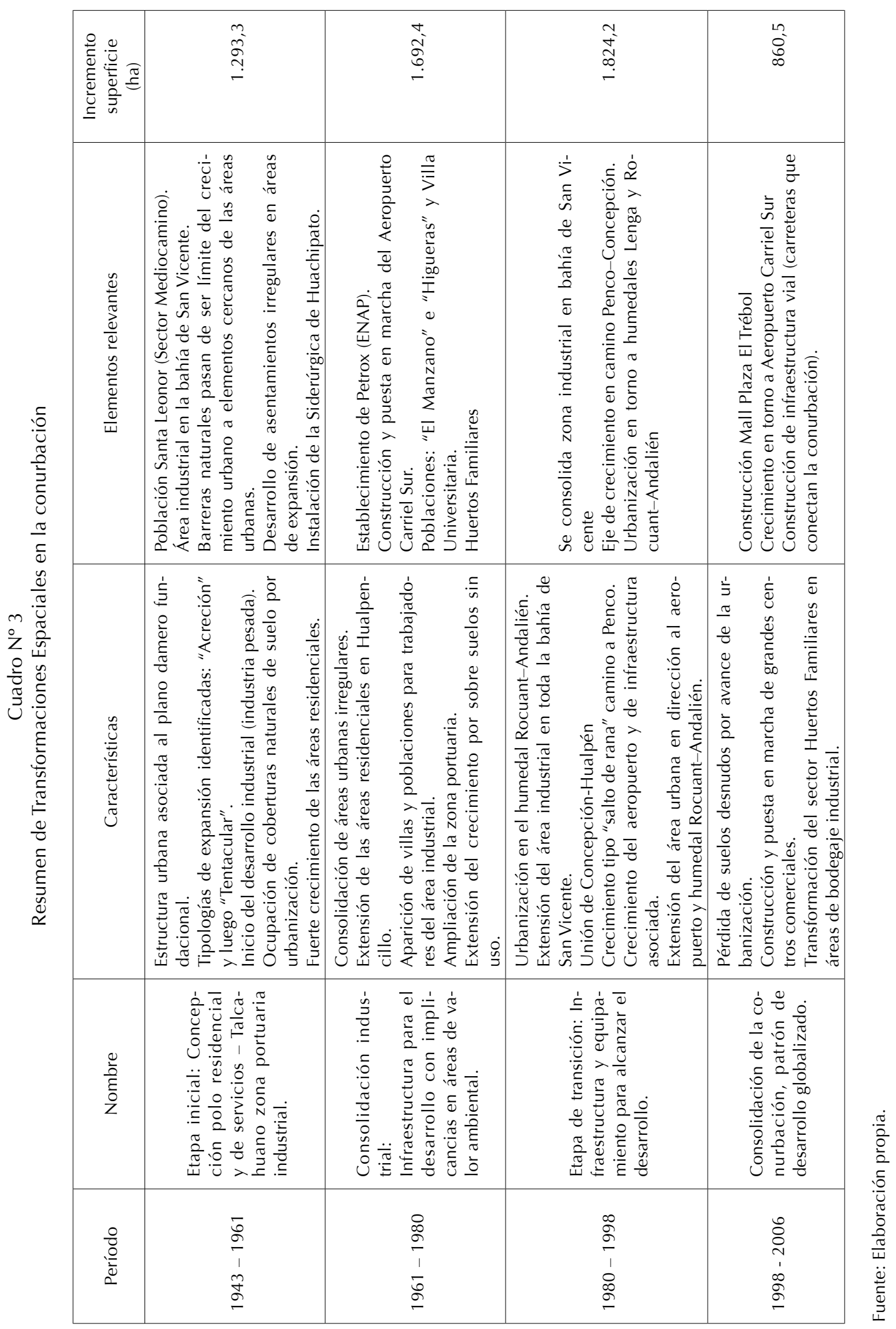




\section{Cambios de uso de suelo asociados al crecimiento urbano}

Es posible identificar ciertas características y elementos representativos del proceso de expansión urbana a lo largo del período analizado, distinguiéndose algunos hechos relevantes que se resumen en la Cuadro $\mathrm{N}^{\circ} 3$. Lo relevante de este proceso es que la ciudad parte de patrones compactos (crecimiento por "acreción"), para luego consolidarse a través de los ejes de vialidad (crecimiento "tentacular") para, en tiempos recientes, tender al patrón característico de ciudad difusa ("salto de rana"), en que los límites urbanos se tornan imprecisos.

Con respecto a los cambios de uso y coberturas de suelo, es importante señalar que el crecimiento urbano residencial se produjo fundamentalmente sobre suelos que previamente se encontraban sin un uso productivo previo (suelos con cobertura herbácea que no correspondía ni a praderas ni a cultivos de ningún tipo, catalogados en esta investigación como "suelos desnudos"). Para graficar momentos relevantes asociados a las transformaciones espaciales, se han seleccionado 3 años representativos del período analizado: $1955 ; 1980$ y 2006.

\section{a) Año 1955}

En la Figura $N^{\circ} 4$ se observa la situación para el año $1955^{13}$. Es importante destacar que el uso mayoritario es el "espacios naturales y/o verdes" que reflejan las áreas no urbanizadas aún en aquella fecha. Los conjuntos de viviendas ya son relevantes aunque no masivos. Las áreas de mayor valor ambiental (humedales, bosques, suelos agrícolas) no habían sido urbanizadas aún, aunque en esa época no poseían ni valor ambiental ni tam-

\footnotetext{
13 La información de 1955 se fotointerpretó sobre la base del área urbana efectiva al año 2006.
}
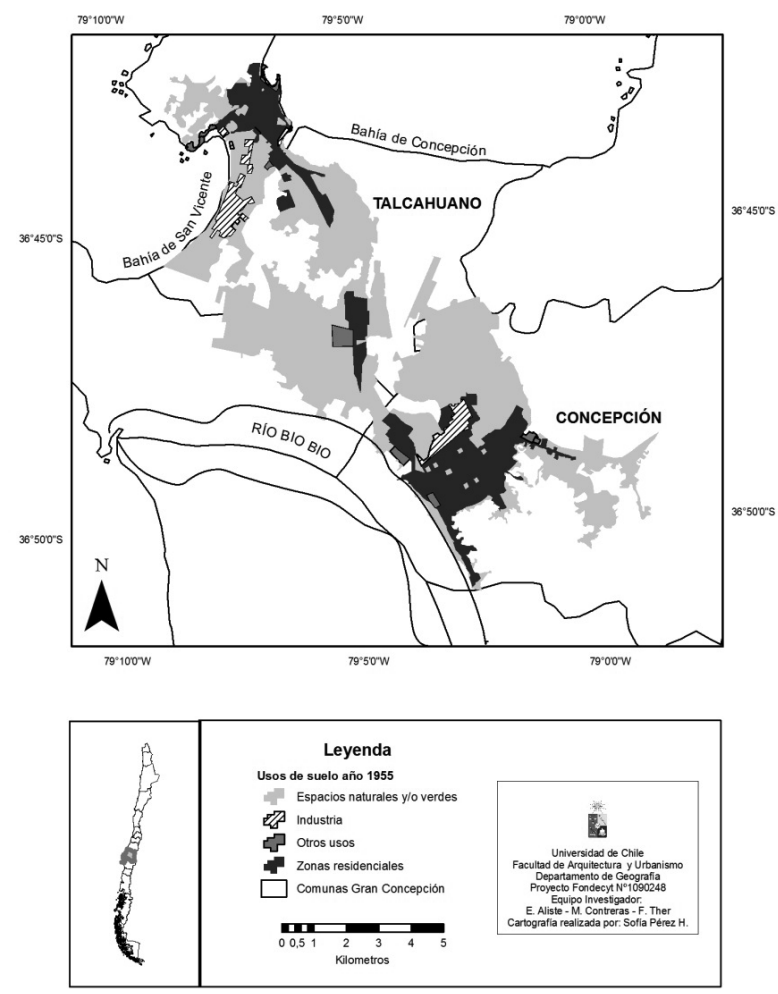

Fuente: Elaboración propia. 
poco la denominación de humedal, pues solo se trataba de pantanos y marismas.

\section{b) Año 1980}

La conurbación de Concepción-Talcahuano comienza a extenderse y a ocupar tierras que hoy son consideradas como zonas importantes para la conservación de la biodiversidad (Figura $N^{\circ}$ 5). A la época, sin embargo, lo interesante es que la ciudad comienza a crecer y extenderse sobre las Ilamadas tierras inútiles e improductivas. Estos cambios en el área de estudio, permiten reflexionar con respecto a lo señalado por Smith y Romero (2009), que afirman que existe un valor que la sociedad asigna a las actividades humanas en desmedro del valor que le entrega al medio natural, pues tiende a inclinarse a la ocupación de estos espacios por industrias o proyectos inmobiliarios, motivada por la generación de recursos económicos de corto plazo. Este hecho no toma en consideración el daño efectuado al medio ambiente en el mediano y largo plazo, con las correspondientes consecuencias negativas sobre la misma sociedad. Sin embargo, conviene señalar que las decisiones de la sociedad en este sentido deben ser miradas y examinadas en perspectiva y en su sentido geohistórico, esto es, considerando que las decisiones de un momento determinado, se hacen en virtud de los conocimientos, necesidades y prioridades de una sociedad en proceso de construcción.

c) Año 2006

Para el año 2006, los usos residenciales se apropiaron extensamente de los suelos con valor natural. Del mismo modo, los usos industriales (sobre todo la industria pesada)

Figura $\mathrm{N}^{\circ} 5$

Usos y coberturas de suelo presentes al año 1980
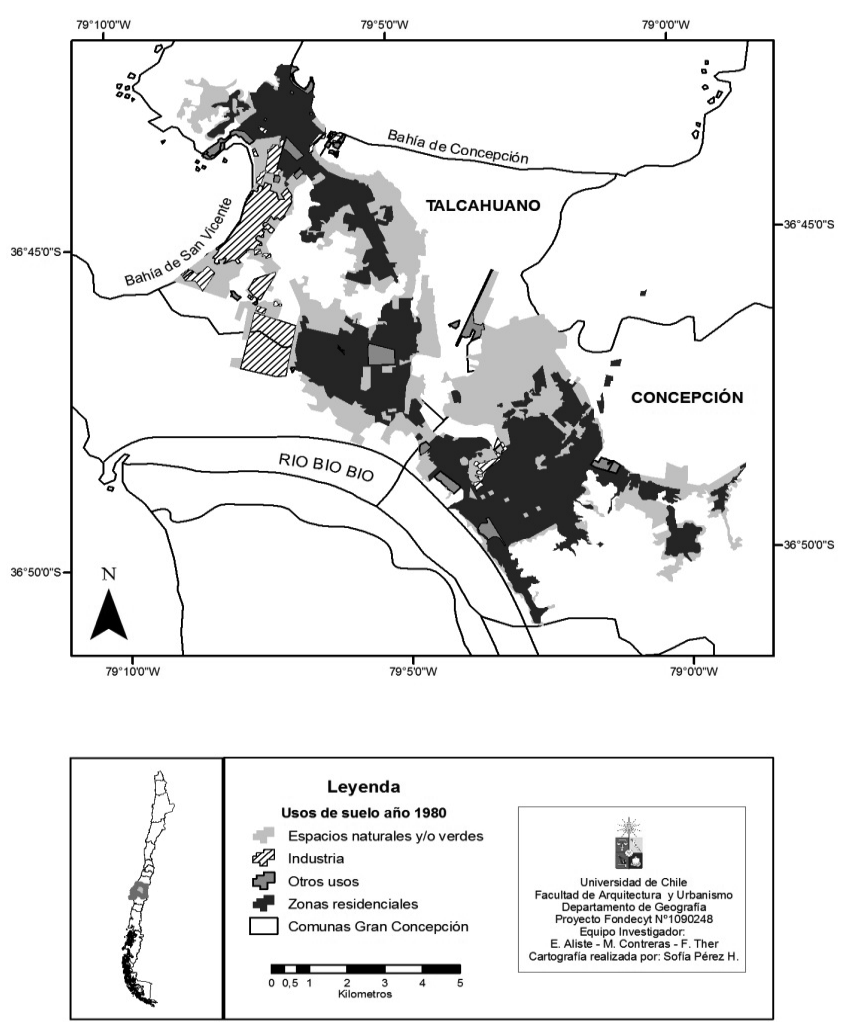

Fuente: Elaboración propia. 
se desarrollaron con mayor fuerza, principalmente en torno a la bahía de San Vicente.

En la Figura $N^{\circ} 6$ se pueden inferir grandes transformaciones ambientales recientes expresadas en la gran presión que ejercen los usos de suelo urbano sobre las áreas con valor natural. Se observa, también, que el desarrollo industrial (industria pesada) se ha extendido en la conurbación con el aumento aproximado de 422,6 hectáreas desde 1980 al 2006. El sector industrial y su relevancia dentro del desarrollo del sistema urbano, permitió que se estableciera una zona asociada a este uso exclusivo en la comuna de Talcahuano, lo que no representa un consumo de suelo tan intenso como el uso residencial, debido a que los sectores industriales y portuarios se desarrollan de acuerdo al crecimiento "encapsulado", es decir, circunscrito a ciertas áreas con destino industrial, tales como par- ques o recintos productivos, que cuentan con reservas para su crecimiento, lo que se traduce en un incremento de suelo muy discreto (Baeriswyl, 2009).

Con respecto de a la evolución de los usos de suelo no urbanos, se destacan importantes cambios, como la pérdida importante de parte de los humedales que existían dentro de los límites de expansión del año 2006, los cuales fueron completamente urbanizados.

Cabe señalar que lo interesante del período desde 1955 al 2006, es que muestra una serie de tendencias que no son otra cosa que la consolidación del modelo de desarrollo impulsado desde la década de 1950 y fuertemente marcados por la idea del crecimiento. No obstante lo anterior, la llegada en las décadas de 1980 y 1990 del discurso de la sustentabilidad, del desarrollo sustentable como asimismo el

Figura $\mathrm{N}^{\circ} 6$

Usos y coberturas de suelo presentes al año 2006

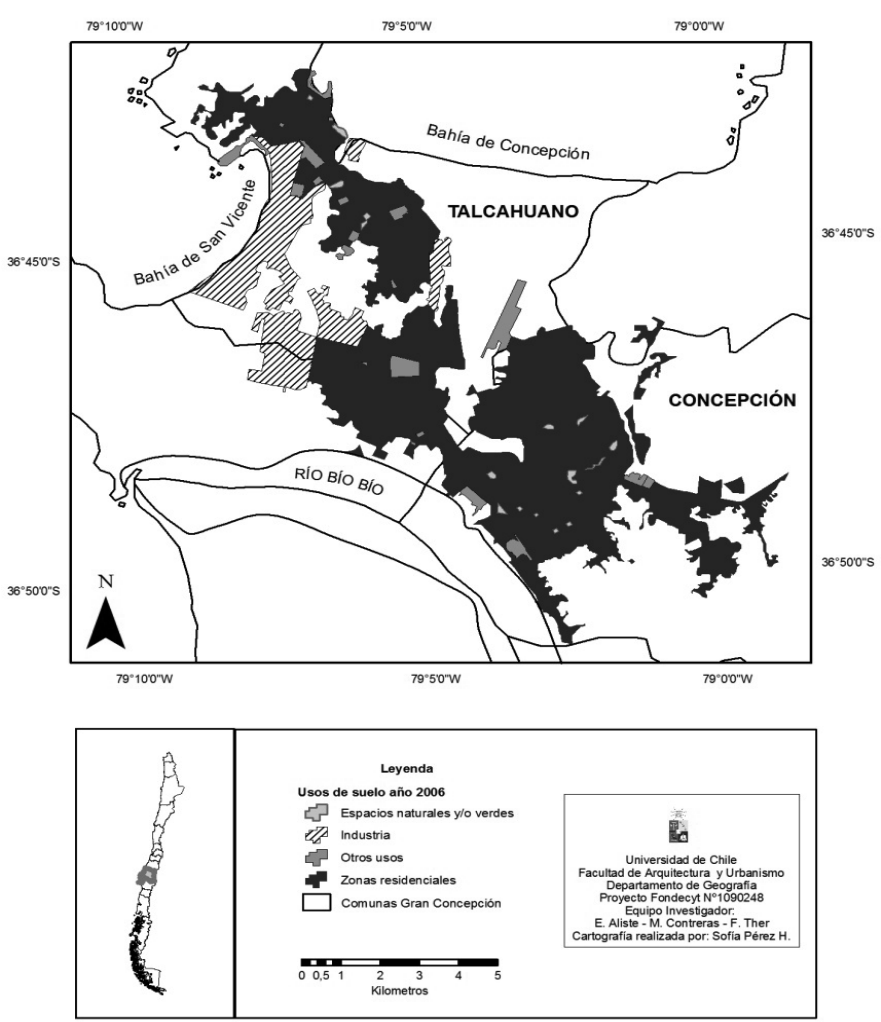

Fuente: Elaboración propia. 
arribo de nuevos conocimientos de carácter ambiental, dejan en evidencia una serie de dificultades y contradicciones que hoy podemos observar a la luz del seguimiento paulatino de este proceso en el tiempo.

Entre las preguntas que hoy caben a este proceso, están, sin lugar a duda, aquellas que relacionan la idea del crecimiento urbano, la estructura económica y las nociones que se acompañan a las ideas de progreso y desarrollo. Lo anterior, en definitiva, resulta clave para acceder a los argumentos y elementos que van incidiendo en la configuración de la geografía social de la ciudad, en tanto es al mismo tiempo la ciudad que se va forjando y creando, con sus reparos y virtudes. En síntesis, la impronta de la ciudad se va marcando y delineando también con esta noción de ciudad industrial.

\section{Discusión y conclusiones}

Los resultados expuestos antes, indudablemente, son provocadores e invitan a pensar la ciudad desde su condición dinámica. En tal sentido, es imprescindible recurrir a las propuestas hechas en este mismo sentido y recientemente por Rojas et al. (2009), Romero et al. (2009) y Romero y Vidal (2010).

No tiene mucho caso, a nuestro juicio, detenerse en precisar y denotar las transformaciones en sí mismas. Lo relevante, creemos, es proponer interpretaciones y desde el plano hermenéutico articular algunas hipótesis respecto de las implicancias ambientales o el nivel de incidencia que estas dinámicas pueden ofrecer en el campo de la sustentabilidad de la ciudad, conforme con los cambios detectados. En definitiva, poco relevante nos parece sostener una crítica al proceso de crecimiento en sí mismo si no se explora de manera más detenida una crítica a las razones, causales $y$, sobre todo, al sentido que hay en la configuración y gestación de una ciudad que hoy carga un sello distintivo e identitario estrechamente vinculado a la industria y el crecimiento urbano. Por lo anterior, la mirada a los discursos ambientales, a la búsqueda de la sustentabilidad urbana y de la planificación en sí misma, requiere una revisión en perspectiva que deje en claro que la situación ambiental de la ciudad, respon- de a condiciones y características históricas bien especiales y precisas, no azarosas y que, por lo demás, son parte de la esencia de la ciudad.

Pese a lo anterior, y a la luz de los nuevos conocimientos más recientes en materias medioambientales, conviene, sin embargo, tener claridad que hay situaciones importantes de destacar.

Las primeras consecuencias que ambientalmente parecen relevantes, comienzan a acusarse en la década de 1980 en la zona de humedales principalmente, con acento en Lenga y Rocuant-Andalién. En el período comprendido entre 1955 y 1980, tal como se ha señalado, se pierde una superficie de poco más de 1.100 hectáreas de humedales, principalmente por uso residencial formal e informal. En este sentido, la fragmentación de áreas con alta calidad ambiental en la zona de los humedales de Rocuant-Andalién, representa una importante pérdida ambiental en áreas valoradas por su contribución a la biodiversidad (Romero et al., 2009).

Junto con lo anterior, la proliferación de diversas industrias en la zona, especialmente en la comuna de Talcahuano (y hoy Hualpén), contribuyó por una parte a un importante deterioro de las condiciones de calidad de las aguas, del suelo y del aire, pero sobre todo a la expansión urbana por demanda de suelo para uso residencial. Ello configuró un panorama ambientalmente muy negativo en la zona, que llevó a impulsar importantes iniciativas con apoyo estatal.

Frente a lo anterior, sin embargo, consideramos la necesidad de enfrentar además otro tipo de cuestiones que busquen complementar lo ya tratado por otros estudios, como algunos de los ya citados. Entre estas, una que nos resulta importante proponer a la luz de nuestros resultados, es la referida a cómo enfrentar la idea de un desarrollo que sea capaz de conciliar la idea de progreso y la referida a la sustentabilidad de las condiciones ambientales del entorno. Lo que podemos desprender del análisis efectuado, es que el discurso del desarrollo y las prácticas espaciales asociadas a estos discursos, van implicando necesariamente modificaciones de carácter radical en las condiciones del 
entorno. De hecho, el momento en que más retroceden los humedales, son precisamente los momentos en que podríamos señalar hay mejores condiciones en cuanto al incremento de las superficies tanto residenciales como industriales. Por una parte los resultados de ciertas acciones de la política económica permiten evidenciar logros importantes en lo que implica el incremento de la infraestructura: más industrias, más viviendas, más carreteras, etc. En nuestros resultados, por el contrario, estos avances en la zona van de la mano con las reducciones de las condiciones de calidad ambiental de los humedales, por señalar un ícono ambiental importante.

En tal sentido, conviene mirar de un modo más amplio aquellos aspectos vinculados a la teoría sobre el desarrollo y mirar en perspectiva los alcances y formas que adquieren en general los postulados sobre la sustentabilidad del desarrollo en la ciudad. Este aspecto se torna esencial en la medida que la "metáfora" del desarrollo en la ciudad, esconde una serie de elementos que pueden mirarse a través de esta suerte de "geohistoria de la industria" en la ciudad. Tal como lo señala Naredo (2006), la mitología del desarroIlo termina ocultando una serie de aspectos al mismo tiempo que se resaltan otros.

En el caso de la ciudad industrial, la mayor parte de las apologías a su contribución al desarrollo son al mismo tiempo las principales contribuciones a su deterioro y radical transformación de manera definitiva. Sin embargo, hay un hecho no menos relevante en este aspecto, y es el que dice relación con la creación y surgimiento, en cada uno de estos momentos, de ciudades distintas; diferentes ciudades son las que surgen en cada una de estas transformaciones, en tanto en cada momento van incorporando también a nuevos actores de estos espacios vividos que, por lo mismo, generan nuevas valoraciones de su medio ambiente (Robbins, 2003; Zimmerer \& Bassett, 2003). A nuestro juicio, lo que acusa este derrotero de transformaciones del ambiente, es al mismo tiempo una importante transformación no solo de las características físico-naturales y estructurales, sino, al mismo tiempo, emerge una ciudad con un sentido diferente $y$, por lo tanto, son actores y a la vez habitantes, que van construyendo una nueva forma de relacionarse con ella. La ciudad puerto, la ciudad de las sociedades comerciales vinculada a la actividad portuaria de la primera mitad del siglo XX (Mazzei De Grazia, 1990), la misma que da paso a la ciudad industrial (Aliste y Almendras, 2010), es luego la ciudad de los servicios de la actualidad (Sandoval, 2010).

Cabe entonces como corolario la pregunta: ¿qué es la ciudad?

\section{Referencias bibliográficas}

ALISTE, E. y ALMENDRAS, A. Trayectoria territorial de la conurbación ConcepciónTalcahuano: industria, asentamientos humanos y expresión espacial del desarrollo, 1950-2000. En: PÉREZ, L. e HIDALGO, R. (editores). Concepción Metropolitano. Evolución y desafíos. Santiago de Chile: Pontificia Universidad Católica de Chile, Serie GEOlibros y Centro EULA, Universidad de Concepción, 2010, p. 123-149.

BAERISWYL, S. Crecimiento urbano del Gran Concepción, patrones y tendencias de una metropolización. En: HIDALGO, R.; DE MATTOS, C. y ARENAS, F. (editores.). Chile: Del país urbano al país metropolitano. Santiago de Chile: Pontificia Universidad Católica de Chile, Serie GeoLibros, 2009, p. 241-255.

BÄHR, J., \& G. MERTINS. Urbanization in Latin America. Applied Geography, 1993, № 41, p. 89-109.

BORSDORF, A. Hacia la ciudad fragmentada. Tempranas estructuras segregadas en la ciudad latinoamericana. Scripta Nova. Revista electrónica de geografía y ciencias sociales, 2003, Vol. VII, No 146 (122). Disponible en Internet: http://www.ub.es/geocrit/sn/sn146(122).htm

CAPEL, H. Morfología de las ciudades. Barcelona: Ediciones del Serbal, 2002.

CHUVIECO, E. Teledetección Ambiental: la observación de la tierra desde el espacio. Barcelona: Editorial Ariel, 2002.

HERNÁNDEZ, H. El Gran Concepción: Desarrollo histórico y estructura urbana. Revista Informaciones Geográficas, 1983, № 30, p. 47-70. 
JANOSCHKA, M. Urbanizaciones privados en Buenos Aires: ¿Hacia un nuevo modelo de ciudad latinoamericana? En: CABRALES BARAJAS, L.F. (editor). Latinoamérica: países abiertos, ciudades cerradas. Guadalajara: Universidad de Guadalajara - UNESCO, 2002, p. 287-318.

MAZZEI DE GRAZIA, L. Sociedades comerciales e industriales y economía de Concepción, 1920-1939. Santiago de Chile: Editorial Universitaria, 1990.

MAZZEI DE GRAZIA, L. y PACHECO, A. Historia del traslado de la ciudad de Concepción. Concepción: Editorial Universidad de Concepción, 1985.

MOSCOSO, C. Cambios en el uso y coberturas de uso y sus efectos sobre la escorrentía urbana. Valparaíso y Viña del Mar período 1980 - 2005. Santiago de Chile: Memoria para optar al título de Geógrafo, Universidad de Chile, 2007 (Inédito).

NAREDO, J.M. Raíces económicas del deterioro ecológico y social. Madrid: Siglo XXI Editores, 2006.

PÉREZ, L. e HIDALGO, R. (editores). Concepción metropolitano. Evolución y desafíos. Santiago de Chile: Serie GEOlibros-Instituto de Geografía, Pontificia Universidad Católica de (hile) y Facultad de Arquitectura y Urbanismo-Centro EULA, Universidad de Concepción, 2010.

ROBBINS, P. Fixed categories in a portable landscape. The causes and consequences of land cover categorization. In: ZIMMERER, K. \& BASSETT, T. Political ecology. An integrative approach to geography and environment-development studies. New York: The Guilford Press, 2003, p. 181-200.

ROJAS, C.; OPAZO, S. y JAQUE, E. Dinámica y patrones de crecimiento urbano del Área Metropolitana de Concepción. Tendencias de las últimas décadas. En: HIDALGO, R.; DE MATTOS, C. y ARENAS, F. (editores). Chile: Del país urbano al país metropolitano. Santiago de Chile: Serie GEOlibros-Instituto de Geografía-Instituto de Estudios Urbanos y
Territoriales, Pontificia Universidad Católica de Chile, 2009, p. 257-268.

ROMERO, H. y SARRICOLEA, P. Patrones y factores de crecimiento espacial de la ciudad de Santiago de Chile y sus efectos en la generación de islas de calor urbanas de superficie. Santiago de Chile: Biblioteca Digital, Universidad de Chile, 2006.

ROMERO, H.; AZÓCAR, G.; FERNANDO, O.; VÁSQUEZ, A. y TOLEDO, X. Ecología urbana y gestión sustentable de las ciudades intermedias chilenas. Revista Medio Ambiente y Desarrollo, 2001, Vol. 17, №4, p. 45-51.

ROMERO, H.; MOSCOSO, C. y SMITH, P. Lecciones y conclusiones sobre la falta de sustentabilidad ambiental del crecimiento espacial de las ciudades chilenas. En: HIDALGO, R.; DE MATTOS, C. y ARENAS, F. (editores). Chile: Del país urbano al país metropolitano. Santiago de Chile: Serie GEOlibros-Instituto de Geografía-Instituto de Estudios Urbanos y Territoriales, Pontificia Universidad Católica de Chile, 2009, p. 89-108.

SANDOVAL, V. Transformaciones sociodemográficas y espaciales en el Gran Concepción, durante la segunda mitad del siglo XX. Santiago de Chile: Memoria para optar al título de Geógrafo, Universidad de Chile, 2010 (Inédito).

SANHUEZA, R. y AZÓCAR, G. Transformaciones ambientales provocadas por los cambios económicos de la segunda mitad del siglo XIX; provincia de Concepción. Revista Geográfica de Chile Terra Australis, 2000, № 45, p. 181-194.

SMITH, P. Evolución espacial y temporal de la calidad ambiental del paisaje de los humedales de Concepción entre 1975 y 2006. Santiago de Chile: Memoria para optar al título de Geógrafo, Universidad de Chile, 2007 (Inédito).

ZIMMERER, K. \& T. BASSETT. Approaching political ecology: Society, nature and scale in human-environment studies. In: Political ecology. An integrative approach to geography and environment-development studies. New York: The Guilford Press, 2003, p. 1-25. 